\title{
LA EDUCACIÓN Y EL ENFOQUE DE GÉNERO EN LA NORMATIVIDAD NACIONAL Y EN EL MUNICIPIO DE MEDELLÍN"
}

\author{
Gustavo Adolfo García Arango** \\ Recibido: Abril 11 de 2014 \\ Aprobado: Mayo 31 de 2014
}

\section{RESUMEN}

El artículo da cuenta de la regulación jurídica nacional en materia de género desde la perspectiva educativa y responde en qué medida se ha regulado jurídicamente la educación en género en el Municipio de Medellín. El proyecto de investigación siguió el método cualitativo a partir de técnicas de análisis documental, a partir de las cuales se realizó la consulta de normas y jurisprudencia a nivel nacional y de los Acuerdos Municipales relacionados en materia educativa. Se encontró que no existe una disposición legal que obligue a los establecimientos educativos a tener una educación diferenciada por sexos o mixta; y se identificaron 14 acuerdos municipales relacionados con el tema. Se concluyó que efectivamente el Concejo de la ciudad ha regulado el tema en diferentes direcciones: en los acuerdos que no versan sobre educación pero tienen un lenguaje incluyente o incluyen a la Secretaría de Educación desde una perspectiva de transversalidad, los acuerdos sobre educación que usan un lenguaje incluyente y los que hacen expresa referencia a la educación de género. Ello evidencia el interés de la Corporación Municipal por regular el tema en la ciudad, pero además en las instituciones educativas, buscando una educación inclusiva, respetuosa, pacífica.

\footnotetext{
* Artículo derivado del proyecto de investigación denominado "estado del arte de los acuerdos municipales de Medellín en materia de educación: periodo 1946-2012”, financiado por la Universidad Católica de Oriente, Rionegro (Antioquia), Grupo de Investigaciones Jurídicas. El autor fue el investigador principal. ** Filósofo, abogado, magíster en derecho Universidad Pontificia Bolivariana. Docente de la Universidad de Antioquia, investigador de la Universidad Católica de Oriente-Grupo de Investigaciones Jurídicas, abogado de la Secretaría de Educación del Municipio de Medellín. Correo electrónico: garcia.arango@yahoo.com
} 
Palabras claves: Acuerdos municipales, derecho educativo, educación mixta, enfoque género, Municipio de Medellín.

\title{
EDUCATION AND GENDER FOCUS WITHIN THE NATIONAL LEGISLATION AND THE MUNICIPALITY OF MEDELLÍN
}

\begin{abstract}
This article gives an account of the national legal regulation of gender from an educational approach and to what extent gender issues in education have been legally regulated in the Municipality of Medellín. The project was carried out according to the qualitative method and the documentary analysis in order to find out national legislation, jurisprudence and Municipal Agreements related to educational matters. Findings point out that there is no legal disposition that demands either single or mixed - gender education from schools, and 14 Municipal Agreements have been identified in relation with this this subject. It is possible to conclude that the City Council has regulated the topic in different directions: agreements that do not refer to education but have an inclusive language or include the Secretary of Education from a transversal perspective, agreements on education that use an inclusive language, and those that especifically refer to gender education. This shows the interest of the Municipal Corporation to regulate the topic not only in the city, but also in educational institutions, looking for an inclusive, respectful and peaceful education.
\end{abstract}

Key words: Municipal agreements, education law, mixed education, gender focus, Municipality of Medellín.

\section{EDUCAÇÃO E GÊNERO NOS REGULAMENTOS NACIONAIS E NO MUNICÍPIO DE MEDELLIN}

\section{RESUMO}

$\mathrm{O}$ artigo relata sobre o sexo regulação jurídica nacional a partir de uma perspectiva educacional e responde ao que medida legalmente regulamentada educação de gênero no Município de Medellín. O projeto de investigação seguiu o método qualitativo com a técnica de documentário, em que as regras de consulta e jurisprudência tornou-se 
nacionalmente e Municipal acordos relacionados no nível de educação. Verifica-se que não há previsão legal exigindo que as instituições de ensino ter diferenciado ou a educação de ambos os sexos; e 14 acordos municipais relacionados ao tema foram identificados. Conclui-se que na verdade a prefeitura cobriu o tópico em diferentes direções: nos acordos não são sobre educação, mas tem uma linguagem inclusiva ou incluir o Departamento de Educação a partir da perspectiva de integração, acordos sobre a educação através de uma linguagem inclusiva e de fazer referência explícita à educação de gênero. Isso mostra o interesse da Corporação Municipal de regular a questão na cidade, mas também nas instituições de ensino, buscando uma educação inclusiva, respeitosa e pacífica.

Palavras chaves: Acordos municipais, direito educacional, educação mista, foco gênero, Medellin.

\section{INTRODUCCIÓN}

La educación es contemplada como un derecho fundamental desde la misma Constitución Política de Colombia (art. 44). Así mismo, tanto el art. 44 como el 45 hacen referencia al desarrollo armónico y la formación integral de niños y adolescentes.

Por su parte, la Declaración de los Derechos de los Niños afirma que es derecho de los niños, recibir educación gratuita y obligatoria, por lo menos en las etapas iniciales. Esta educación deberá favorecer "su cultura general y le permita, en condiciones de igualdad de oportunidades, desarrollar sus aptitudes y su juicio individual, su sentido de responsabilidad moral y social, y llegar a ser un miembro útil de la sociedad" (Principio 7).

Por otro lado, la Convención sobre los Derechos del Niño señala que los Estados que hacen parte de la Convención reconocen el derecho a la educación (art. 28.1) y que convienen que debe estar orientada a "preparar al niño para asumir una vida responsable en una sociedad libre, con espíritu de comprensión, paz, tolerancia, igualdad de los sexos y amistad entre todos los pueblos" (literal d, Art. 29.1).

Hoy en día, hacer referencia a los niños es un hecho global que encierra los derechos tanto de varones como de mujeres sin mucha discusión, 
aunque en la realidad se concentren algunas prácticas discriminatorias que han sido sancionadas no solo por los grupos sociales sino desde el mismo sistema jurídico, tanto nacional como internacional.

En ese avance y verificación del estado del arte de las normas en materia de educación, surge la necesidad de realizar un rastreo que determine en qué medida se ha regulado jurídicamente la educación en género en el Municipio de Medellín.

Por ello, sin el interés de hacer una discusión conceptual ni teórica sobre el género, se desarrolló la investigación desde el marco jurídico respondiendo la pregunta: ¿En qué medida se ha regulado jurídicamente la educación en relación al género en el Municipio de Medellín? Situación que no había sido abarcada por la Unidad Jurídica de la Secretaría de Educación ni la Secretaría de las Mujeres de la ciudad.

Por lo anterior se identificó la necesidad de establecer qué normas se han expedido en el Municipio de Medellín sobre la educación en relación al género. Dado que en 1940 se constituyó lo que hoy es la Secretaría de Educación, se optó por realizar el rastreo normativo desde el año 1943 hasta el año 2012.

Los fines que la investigación ha perseguido están enmarcados en objetivos exploratorios y descriptivos, alrededor de los cuales se buscó establecer si existían normas nacionales que determinaran una educación mixta, identificar los acuerdos municipales expedidos por el Concejo que tuvieran relación con el género y establecer en qué medida se ha regulado la educación en relación al género en el Municipio de Medellín.

Respecto de la estrategia metodológica, el proyecto de investigación siguió el método cualitativo con técnica documental, bajo el cual se hizo la consulta de los Acuerdos Municipales relacionados en materia educativa directamente en la Gaceta Oficial del Municipio de Medellín en el Centro de Documentación y página Web oficial del Concejo de Medellín. El rastreo tuvo dos momentos: recolección y validación de la información. En el primero, dos auxiliares de investigación realizaron la búsqueda de manera independiente y en momentos diferentes. Una vez entregado el material recopilado se procedió a confrontar las normas encontradas y a unificar la información para lograr una sola base complementada. 
La validación se hizo en dos fases: en la primera, se hizo un tercer rastreo con una auxiliar adicional; en la segunda, se hizo un cruce de información con base en los listados de Acuerdos que ya poseía la Secretaría de Educación. De las normas compiladas se establecieron los tópicos, uno de los cuales fue el género en la educación, alrededor del cual se hicieron los análisis y el desarrollo del informe final de la investigación. En relación con el enfoque de género salen los siguientes resultados, los cuales son previamente contextualizados en el marco jurídico del derecho educativo de orden nacional para lograr entender, en parte, el proceso normativo ocurrido en el Municipio de Medellín en la relación educación-género.

\section{NO HAY DISPOSICIÓN JURÍDICA QUE OBLIGUE A QUE TODAINSTITUCIÓN SEA MIXTA}

Del rastreo normativo sobre el tema, se encontró que por Ley 12 de 1886 se dispuso sobre la instrucción pública. Dicha ley fue reglamentada por el Decreto 595 de 1886, el cual estipulaba que el sostenimiento de las escuelas urbanas de niñas estaba a cargo de los Departamentos (art. 121).

Así mismo, se hacía referencia a la educación por sexos, al señalar en el art. 126 que los habitantes de cada distrito educativo estaban obligados a sostener las escuelas primarias que fueran necesarias para que las niñas de siete a quince años de edad pudieran recibir educación gratuita.

El artículo 132 estipulaba que las escuelas de niñas debían ser administradas por "señoras de notoria respetabilidad y buena conducta" y que los distritos en los cuales no hubiera sido posible abrir escuelas para varones y para niñas de manera separada por falta de alumnos, quedaba a criterio del Inspector Provincial establecer escuelas alternadas en los dos sexos (art. 132), es decir, que atendían a los hombres y las mujeres en jornadas o días diferentes.

Por ejemplo, Chavarría Serna (2014) señala que desde la Secretaría de Instrucción Pública de Antioquia se expidió el Decreto 01 de 1894, el cual reguló los horarios de clase para las Escuelas Alternadas: de 6 a.m. a 10 a.m. para los varones, y de 11 a.m. a 3 p.m. para las niñas (p. 117).

Posteriormente vino la Ley 0089 del 7 de Noviembre de 1888, sobre instrucción pública nacional, que disponía en el artículo 46: 
Los Municipios que carezcan de recursos suficientes para sostener Escuelas elementales de niñas, podrán ser auxiliados con fondos del Tesoro nacional, a juicio del Gobierno; y para esto se destinan hasta treinta mil pesos anuales, que se incluirán en los respectivos Presupuestos.

En 1892 se expide una nueva ley de instrucción pública primaria, reglamentada por el Decreto 0429 de 1893, la cual deja textualmente en los arts. 14 y 15 lo dispuesto por el Decreto anterior en los arts. $126 \mathrm{y}$ 132.

Más adelante, es expedida la Ley 0039 de Octubre 26 de 1903, también sobre instrucción pública, en la que se estable que "en cada una de las ciudades capitales de los Departamentos existirá una Escuela Normal para varones y otra para mujeres..." (Art. 13). Esta ley fue reglamentada por el Decreto Nacional 0491 del 3 de junio de 1904, que replicaba lo dispuesto en los arts. 14 y 15 del Decreto 0429 de 1893.

La educación, como puede verse, era marcadamente sexista, en el sentido de que discriminaba la educación por sexos, situación que puede tener otra lectura: "El orden social construido de manera sexista contra las mujeres se mantuvo de modo eficaz gracias a la educación diferenciada para niñas y niños. A cada sexo se le transmitían en exclusiva los valores humanos considerados adecuados para su perpetuación." (Martínez, 2004,p. 57).

Esta situación desde la educación, trató de corregirse desde la Convención sobre la eliminación de todas las formas de discriminación contra la mujer, adoptada por la Asamblea General de las Naciones Unidas en 1979 y firmada en Copenhague en 1980. En Colombia esta Convención fue aprobada mediante la Ley 51 de 1981.

En el art. 10 de la Convención se desarrolla todo lo relacionado con la igualdad de derechos de las mujeres con el hombre en materia de educación y a todos los niveles (enseñanza preescolar, general, técnica y profesional), tanto urbana como rural; igualdad de oportunidad de acceso a la educación general y complementaria, así como obtención de becas.

Concretamente en relación con la educación mixta, dispone la Convención: 
La eliminación de todo concepto estereotipado de los papeles masculino y femenino en todos los niveles y en todas las formas de enseñanza, mediante el estímulo de la educación mixta y de otros tipos de educación que contribuyan a lograr este objetivo y, en particular, mediante la modificación de los libros y programas escolares y la adaptación de los métodos de enseñanza. (Lit. c, art. 10)

En la actualidad, la Ley General de Educación, Ley 115 de 1994, que hace referencia a los niños como concepto global para el género masculino y femenino, dispone en el artículo 13 que uno de los objetivos comunes a todos los niveles educativos es el "la construcción de la identidad sexual dentro del respeto por la equidad de los sexos, la afectividad, el respeto mutuo y prepararse para una vida familiar armónica y responsable" (literal d), pero no hace referencia expresa a la educación mixta.

El artículo 138 de la Ley General de Educación, expedida en 1994 y vigente en la actualidad, define los establecimientos educativos y no establece dentro de la naturaleza y condiciones que deban ser mixtos, femeninos o masculinos.

Al año siguiente, Colombia aprobó la Convención Internacional para prevenir, sancionar y erradicar la violencia contra la mujer, suscrita en la ciudad de Belem do Pará, Brasil de 1994, por medio de la Ley 248 de 1995, en la cual se hace varias veces mención al tema educativo. El literal b) del artículo 8, dispone que los Estados que forman parte del Convenio deben adoptar programas para

Modificar los patrones socioculturales de conducta de hombres y mujeres, incluyendo el diseño de programas de educación formales y no formales apropiados a todo nivel del proceso educativo, para contrarrestar prejuicios y costumbres y todo otro tipo de prácticas que se basen en la premisa de la inferioridad o superioridad de cualquiera de los géneros o en los papeles estereotipados para el hombre y la mujer que legitimizan o exacerban la violencia contra la mujer...

Luego se expide la Ley 1098 de 2006, "por la cual se expide el Código de la Infancia y la Adolescencia", que incluye un lenguaje diferencial al hacer referencia a los niños, a las niñas y a los adolescentes y hace 
referencia expresa a la perspectiva de género en el artículo 12 al señalar lo siguiente:

Perspectiva de género. Se entiende por perspectiva de género el reconocimiento de las diferencias sociales, biológicas y psicológicas en las relaciones entre las personas según el sexo, la edad, la etnia y el rol que desempeñan en la familia y en el grupo social. Esta perspectiva se debe tener en cuenta en la aplicación de este código, en todos los ámbitos en donde se desenvuelven los niños, las niñas y los adolescentes, para alcanzar la equidad.

Así mismo, dispone en el artículo 203 que uno de los principios rectores de las políticas públicas en infancia, adolescencia y familia es el de la perspectiva de género (numeral 12).

En una dimensión más amplia, en el art. 42, el Código de la Infancia y la Adolescencia establece que una de las obligaciones de las instituciones educativas es la de "evitar cualquier conducta discriminatoria por razones de sexo, etnia, credo, condición socio-económica o cualquier otra que afecte el ejercicio de sus derechos" (núm. 12, art. 42); formar en "la tolerancia hacia las diferencias entre personas" (núm. 1, art. 43); y "orientar a la comunidad educativa para la formación en la salud sexual y reproductiva y la vida en pareja” (núm. 10, art 44).

Pese a todo lo anterior en la actualidad no existe una sola disposición jurídica (ni normativa ni jurisprudencial) que establezca como obligatoria la educación mixta o discriminada por sexo para todos los establecimientos educativos o en una misma institución, lo que permite que las entidades privadas puedan ofrecer una educación especializada para mujeres o que una misma institución tenga una jornada o una sección femenina y otra mixta.

Por ello, se considera que la determinación de la educación para una población femenina, masculina o mixta depende del proyecto educativo institucional de cada establecimiento educativo. Incluso, no existe norma alguna que establezca que todas las jornadas (mañana, tarde, noche), que todas las sedes (principal y secciones) o que todos los niveles (preescolar, básica y media) deban ser mixtos, porque dicha determinación le correspondería a la institución educativa en virtud de la autonomía escolar plasmada en el proyecto educativo institucional-PEI- el cual es construido por la misma comunidad educativa atendiendo las 
condiciones sociales, culturales y económicas del sector (art. 15 Decreto Nacional 1860 de 1994), lo que permitiría tener una oferta educativa para niños en una jornada, niñas en otra y mixta para un tercera.

Así, una institución podría ser femenina o mixta, siempre que esté definido en el proyecto educativo institucional adoptado por la comunidad educativa, en el entendido de que "cada establecimiento educativo goza de autonomía para formular, adoptar y poner en práctica su propio proyecto educativo institucional sin más limitaciones que las definidas por la ley y este reglamento" (art. 15, Decreto 1860 de 1994), teniendo en cuenta siempre el contexto social, cultural, vocacional e histórico de la institución, lo que implica una organización completa desde lo pedagógico y misional respecto de temas como el uso del tiempo libre, la orientación de la media técnica, la cátedra de educación sexual, la infraestructura de los sanitarios, las clases de educación física, el servicio social estudiantil, entre muchos otros temas propios de la educación básica y media.

Por ejemplo, respecto de la infraestructura sanitaria, la Norma Técnica Colombiana NTC 4595 sobre Planeamiento y Diseño de Instalaciones y Ambientes Escolares, dispone:

4.3.4 Los servicios sanitarios se deben calcular por aparatos y áreas como se muestra en la tabla 5. (La expresión "aparato" hace referencia a un sanitario o un orinal más un lavamanos). Se recomienda instalar aparatos sanitarios de bajo consumo (véase la NTC 920-1).

4.3.5 Los baños para Preescolar pueden ser de uso mixto y deben estar situados en el mismo nivel en que se encuentren los ambientes de trabajo, a no más de $20 \mathrm{~m}$ del puesto más lejano. Dentro del área estipulada se prevé la dotación de una ducha por cada 30 niños. Del nivel escolar hacia arriba, incluidos los vestidores, deben existir baterías independientes, las cuales no deben estar ubicadas a más de $50 \mathrm{~m}$ del puesto de trabajo más lejano. En el tipo de Administración y Docencia no podrá existir menos de un aparato por sexo. (Subrayas fuera de texto)

Como se observa, la norma técnica, de obligatorio cumplimiento para la infraestructura escolar, determina baños independientes para hombres y mujeres, y no opera por capricho de la entidad. 
Y así como se presenta la exigencia de unos espacios físicos adecuados a las características propias de cada género, la legislación ha ido dando un giro para el reconocimiento de las necesidades propias de las mujeres, por ello el sistema jurídico colombiano ha establecido una protección especial para las madres cabeza de familia, la ley de cuotas en los cargos directivos del Estado, la obligación de requisa a mujeres por parte de personal femenino, igualdad salarial, entre otras obligaciones jurídicas que han sido avaladas constitucionalmente, no encontrándose en ello una violación al principio de igualdad, sino como estrategia válida para alcanzar la equidad de género.

\section{EDUCACIÓN Y GÉNERO EN EL DERECHO COLOMBIANO}

En la actualidad, en el margen de la Constitución Política, la Ley General de Educación y la jurisprudencia de la Corte Constitucional, el derecho educativo colombiano ha establecido algunas pautas relacionadas con el género y la educación.

\subsection{Educación diferencial, educación incluyente.}

Las discusiones que desde siglos pasados se han presentado sobre la educación mixta en Colombia han desembarcado en el discurso de la educación incluyente, en la que socializan niños y niñas por igual en los mismos espacios y compartiendo los mismos sistemas de educación.

Esta posición es asumida por la doctrina de igual modo:

A mi juicio, uno de los elementos básicos que configuran la educación pública en los países democráticos es la educación mixta o coeducación, la cual es, sin duda, algo más que poner juntos a niñas y niños en las aulas (...) pero empieza por esa presencia mixta del alumnado en las clases. (Martínez, 2004,p. 55)

En Colombia, la Ley 1620 de 2013, "por la cual se crea el sistema nacional de convivencia escolar y formación para el ejercicio de los derechos humanos, la educación para la sexualidad y la prevención y mitigación de la violencia escolar", asume una posición concreta al señalar que uno de los principios del sistema nacional de convivencia escolar y la educación para la sexualidad es la diversidad, que se basa en el reconocimiento, respeto y valoración propia y de los demás, "sin discriminación por razones de género, orientación o identidad sexual, etnia o condición física, social o cultural" (art. 4). 
Pero la palabra género implica una perspectiva psicológica, antropológica y sociológica que exige una amplitud en el marco regulatorio desde el Derecho. Por ello, la Corte Constitucional ha abarcado el tema en varias sentencias y concretamente ha marcado el horizonte desde la jurisprudencia en sentencia de constitucionalidad, justamente al hacer la revisión del proyecto de ley estatutaria sobre estatuto de ciudadanía juvenil:

El concepto género es una creación social que frecuentemente se contrasta con el término "sexo", que se refiere más bien, a las diferencias biológicas entre hombres y mujeres, en este sentido, resulta erróneo identificar o asimilar la palabra género con sexo. El género es una noción explicativa de las relaciones entre los seres humanos más amplia, mientras que la segunda categoría, da cuenta exclusivamente a las diferencias biológicas y fisiológicas entre mujeres y hombres. Asimismo, género no es igual a "mujer" o a "hombre", pues engloba también los roles socio-culturales que se asignan a cada uno de los sexos en la sociedad por el hecho de nacer con atributos femeninos o masculinos. (Sentencia C-682, 2012).

Hoy, dicho proyecto normativo es la Ley 1622 de 2013 y trae una definición legal en los siguientes términos:

Género. Es el conjunto de características, roles, actitudes, valores y símbolos construidos socialmente que reconoce la diversidad y diferencias entre hombres y mujeres en pleno goce o ejercicio de sus derechos y libertades fundamentales, en condiciones de igualdad en las esferas política, económica, social, cultural o en cualquier otra esfera de la vida pública. (N. 5, art. 5).

Consecuente con lo anterior, la norma trae como funciones de los comités municipales de convivencia escolar el fomento del desarrollo de competencias ciudadanas que incluye en los procesos formativos, "la autonomía, la perspectiva de género y el ejercicio de los derechos humanos, sexuales y reproductivos" (N. 1, art. 10).

Y el art. 20 de la misma ley, haciendo referencia a los proyectos pedagógicos de educación para la sexualidad deben incluir, entre otras, las reflexiones en relación con las emociones, la construcción social de 
la sexualidad, "los comportamientos culturales de género, la diversidad sexual, la sexualidad y los estilos de vida sanos, como elementos fundamentales para la construcción del proyecto de vida del estudiante."

En esta misma dirección y teniendo en cuenta que una buena porción de los estudiantes son jóvenes, dentro del marco educativo debe considerarse el estatuto de ciudadanía juvenil, Ley 1622 de 2013, que tiene entre sus finalidades promover las relaciones equitativas entre géneros (N. 5, art. 2); así mismo, dispone que la interpretación que se haga de ella misma debe hacerse desde un enfoque diferencial de género (N. 2, art. 3; numerales 8 y 18, art. 4; art. 6; N. 1, art. 14).

Por su parte, la perspectiva de género la define el Código de la Infancia y la Adolescencia, que igualmente aplica en el espacio escolar, en los siguientes términos:

PERSPECTIVA DE GÉNERO. Se entiende por perspectiva de género el reconocimiento de las diferencias sociales, biológicas y psicológicas en las relaciones entre las personas según el sexo, la edad, la etnia y el rol que desempeñan en la familia y en el grupo social. Esta perspectiva se debe tener en cuenta en la aplicación de este código, en todos los ámbitos en donde se desenvuelven los niños, las niñas y los adolescentes, para alcanzar la equidad. (Art. 12, Ley 1098 de 2006).

En general, puede evidenciarse la clara tendencia al reconocimiento de la diversidad en cuanto al género y su integración en el sistema educativo desde el mismo sistema jurídico nacional.

\subsection{Cátedra de educación sexual.}

Si bien, cuando se habla de género se va mucho más allá de lo meramente sexual, su importancia es innegable, todavía más en el escenario educativo.

...la sexualidad es un fenómeno de enorme complejidad, por cuanto se proyecta en distintas dimensiones. Así, desde el punto de vista social, la sexualidad hace referencia a los diversos papeles que los patrones socio-culturales existentes asignan a los diferentes sexos. Es lo que algunos 
autores denominan los roles de género. Sicológicamente, la sexualidad alude no sólo a la identidad que al respecto se forman los seres humanos, sino que tiene además aspectos comportamentales, ligados a la orientación afectiva que tienen los individuos por personas de determinado sexo. Y finalmente la sexualidad tiene una clara dimensión biológica. (Sentencia de Unificación 337, 1999).

Bajo esa perspectiva general, teniendo en cuenta una educación integral, la educación sexual se presenta como parte fundamental en el desarrollo armónico de los niños, niñas y adolescentes (T-440/92). Por ello, la Ley General de Educación contempla como uno de los objetivos comunes a todos los grados educativos, el desarrollo de una sana sexualidad, la construcción de la identidad sexual y el respeto por la equidad de los sexos (art. 13, Ley 115 de 1994) y la educación sexual se encuentra dentro de las áreas obligatorias transversales (art. 14).

Y el Código de la Infancia y la Adolescencia, respecto de la educación, impone como obligación complementaria de las instituciones educativas, "orientar a la comunidad educativa para la formación en la salud sexual y reproductiva y la vida en pareja" (art. 44, Ley 1098 de 2006).

La reglamentación del tema la hace el Ministerio de Educación Nacional a través de la Resolución 3353 de 1993, "por la cual se establece el desarrollo de programas y proyectos institucionales de Educación Sexual en la Educación básica del país”. Esta norma confirma la obligatoriedad de esta educación en todos los grados educativos, las competencias a lograr, las consideraciones para la organización de los proyectos educativos, las responsabilidades tanto del Ministerio de Educación como de las secretarías de educación, el sistema de evaluación, entre otros, ello sin desconocer que la primera obligada en ofrecer una adecuada educación sexual es la familia (Sentencia T-440, 1992, Sentencia T-458, 2007).

La Corte también estudió las relaciones entre la política pública de educación sexual y el derecho fundamental a la educación en la sentencia T-220 de 2004, confirmando la dimensión integral de la persona humana.

3.3 Mujeres; cabeza de familia y violencia. 
Siendo clara la situación de desventaja de las madres cabeza de familia, la Ley 82 de 1993, por la cual se expiden normas para apoyar de manera especial a la mujer cabeza de familia, modificada por la Ley 1232 de 2008, hace varias referencias al aspecto educativo, al establecer que el Gobierno Nacional debe garantizarles el acceso a la educación básica, media y superior, con cobertura, calidad y pertinencia (art. 3), por ello se autoriza a los municipios establecer acceso preferencial a los auxilios educativos (art. 9) y servicio básico de textos (art. 5 y 9); así mismo, establece un tratamiento preferencial para el acceso al sistema educativo de los hijos de las mujeres cabeza de familia (art. 7).

En relación con a la violencia contra las mujeres el Congreso de la República a través de la Ley 1257 de 2008, por la cual se dictan normas de sensibilización, prevención y sanción de formas de violencia y discriminación contra las mujeres, se reforman los Códigos Penal, de Procedimiento Penal, la Ley 294 de 1996 y se dictan otras disposiciones, dispuso un paquete de medidas educativas contenidas en el art. 11, en el cual asignó como funciones del Ministerio de Educación Nacional, "velar para que las instituciones educativas incorporen la formación en el respeto de los derechos, libertades, autonomía e igualdad entre hombres y mujeres como parte de la cátedra en Derechos Humanos", así como "desarrollar políticas y programas que contribuyan a sensibilizar, capacitar y entrenar a la comunidad educativa, especialmente docentes, estudiantes y padres de familia, en el tema de la violencia contra las mujeres." Además, le encomendó la misión de implementar medidas para mantener en el sistema educativo a las mujeres víctimas de la violencia, evitando su desescolarización.

\subsection{Estudiantes embarazadas.}

Una de las prácticas educativas que tuvo que entrar a resolver la Corte Constitucional en su ejercicio de protección de los derechos fundamentales, fue en relación con los derechos de las estudiantes embarazadas, situación que presentaba conflictos morales y disciplinarios en el marco de los manuales de convivencia de los establecimientos educativos.

En múltiples fallos (Sentencia T-656,98, Sentencia T-393,09, Sentencia T-564,09, Sentencia T-603,13) la Corte ha revisado casos en los cuales se han visto sancionadas las estudiantes que han quedado embarazadas o a las que incluso se les ha negado un cupo por su estado (T-290/96, T412/99). En sus pronunciamientos la Corporación ha dispuesto que, "El 
embarazo de una estudiante no es una situación que pueda limitar o restringir su derecho a la educación, por lo que, ni los manuales de convivencia de las instituciones educativas, ni el reglamento interno, pueden, ni explícita, ni implícitamente, tipificar negativamente el estado de gestación de una alumna”.(Sentencia T-396, 2009).

La Corte ha enfatizado en el derecho a la maternidad y a la educación en el marco de la perspectiva ética y religiosa, propia particularmente de los establecimientos educativos privados:

La protección que el Estatuto Superior depara a la maternidad es de tal intensidad que ni siquiera aquellos centros educativos cuyo proyecto de educación se encuentre fundado en una determinada visión ética $\mathrm{o}$ religiosa del mundo - protegida por la libertad de conciencia - pueden utilizar tal visión para estigmatizar, apartar o discriminar a una estudiante en estado de embarazo de los beneficios derivados del derecho a la educación. En otras palabras, ante la tensión que puede existir entre la autonomía de los centros privados de educación y el derecho de la futura madre a no ser discriminada por razón de su embarazo, prima, sin duda, este último. (Sentencia T$656,1998)$.

En conclusión, vía jurisprudencial, las madres gestantes tienen doble protección en el sistema educativo, tanto por el derecho a la maternidad como por el derecho a la educación, lo que obliga a las directivas de los establecimientos educativos tanto oficiales como privados (incluso los que profesan algún tipo de credo) a garantizar la permanencia escolar de las madres, cosa que pueda implicar modificar sus respectivos manuales de convivencia.

\subsection{Identidad de género.}

Respecto de las orientaciones sexuales e identidades de género de los estudiantes en el marco educativo, la construcción jurídica ha sido por vía jurisprudencial, principalmente de la Corte Constitucional, que en varias sentencias de tutela ha abarcado la tensión que se presenta entre los manuales de convivencia (principal norma dentro de los establecimientos educativos en el país) y los derechos de los niños, niñas y adolescentes. 
En la sentencia T-565/13, la Corte revisó el caso de un adolescente de 15 años que "se reconoció con una identidad sexual diversa y, por ende, decidió llevar el pelo largo conforme con el género femenino", situación que derivó en una sanción de suspensión por dos días, al no acatar lo dispuesto por el manual de convivencia. Como guía metodológica, la Corte plantea el problema jurídico en los siguientes términos: “¿Se vulneran los derechos fundamentales al libre desarrollo de la personalidad, la igualdad y la identidad sexual o de género, cuando una institución educativa impone sanciones a un alumno adolescente, quien opta por portar el pelo largo y usar maquillaje, en razón de reconocerse con una identidad sexual diversa?"

El Tribunal Constitucional responde expresando, entre otros razonamientos, que los establecimientos educativos tienen prohibido imponer a los estudiantes una apariencia física basada en un modelo que se considera arbitrariamente como deseable o, menos aún, normal, puesto que ello afecta el libre desarrollo de la personalidad de los y las estudiantes, y también "se opone a un ejercicio educativo comprometido, desde la Constitución, con el pluralismo y el respeto a la diferencia"; que, además, la apariencia está vinculada al derecho de la identidad sexual y de género. (Sentencia T-565, 2013).

En este último sentido, afirma que por vía jurisprudencial se ha confirmado que "el reconocimiento de la diversidad en la identidad sexual y de género es uno de los objetivos del proceso educativo que (...) está basado en la promoción del respeto a la diferencia y la promoción de una perspectiva pluralista y democrática". (Sentencia T$656,2013)$.

Así lo había expresado la Corte en la sentencia SU-641/98, cuando manifestó que el género al que pertenece el adolescente, la opción sexual y sus características físicas no pueden ser factor ni de sanción ni de exclusión, "aunque sí pueden ser factor a tener en cuenta para la especialización de las instituciones en la educación masculina, femenina o especial..."

En caso anterior, el Alto Tribunal mediante sentencia T-101/98 trató el caso de dos adolescentes a los que les fue negado el cupo por ser homosexuales, en una institución educativa oficial dirigida por una comunidad religiosa católica. En el texto, la Corte tutela el derecho a la educación de ambos estudiantes exigiendo al rector garantizar los cupos correspondientes, señalando que, "(...) la homosexualidad es una 
condición de la persona humana que implica la elección de una opción de vida tan respetable y válida como cualquiera, en la cual el sujeto que la adopta es titular, como cualquier persona, de intereses que se encuentran jurídicamente protegidos, y que no pueden ser objeto de restricción por el hecho de que otras personas no compartan su específico estilo de vida. (Sentencia T-101, 1998).

Así, la identidad y orientación sexual se protegen constitucionalmente, incluso en el campo educativo, toda vez que ellos forman parte del núcleo esencial de la dignidad, la libertad y la autonomía de toda persona. (Sentencia T-062, 2011).

\section{SOBRE LAS COMPETENCIAS DE LOS CONCEJOS EN MATERIA DE EDUCACIÓN}

En 1886 de expide una nueva Constitución Política, que en el artículo 185 estableció que le correspondía "a las Asambleas dirigir y fomentar por medio de ordenanzas y con los recursos propio del Departamento, la instrucción pública..." No obstante, siempre se conservan las competencias municipales en materia de educación. En el artículo 198 estipulaba que en cada distrito municipal habría una corporación popular con el nombre de Consejo Municipal (con "s") pero supeditados en los gastos a las ordenanzas departamentales.

Así lo señala García Estrada (2000):

...la Constitución del 86 atentó, de manera evidente, contra la autonomía municipal dado que la labor de los concejos, y aun la del alcalde, estaba subordinada a las disposiciones departamentales emanadas de la asamblea. Las demás funciones a que se refiere la Constitución regeneradora se fueron perfilando y definiendo, con cierto detalle, a finales del siglo XIX en las diferentes reformas institucionales. En realidad, las atribuciones de los concejos no cambiaron mucho: estaba encargado de la construcción y conservación de los edificios y mobiliarios de las escuelas públicas, del pago de empleados de las escuelas urbanas de niñas y de la mitad del sueldo de los directores de las escuelas alternas; de mantener una escuela de instrucción primaria gratuita; también tenía bajo su responsabilidad los gastos ocasionados por el aprendizaje de artes y oficios en escuelas urbanas... entre otras (p. 43-44). 
El art. 67 de la Constitución Política dispone que el Estado, la sociedad y la familia sean responsables de la educación. Pero que es función del Estado "regular y ejercer la suprema inspección y vigilancia de la educación con el fin de velar por su calidad..." Esto frente al orden nacional, pero también están las obligaciones del Estado a través de las entidades territoriales.

Para el caso, las funciones de los municipios y de los concejos respecto de la educación están en la Ley 115 de 1994, que destina el Título VIII a la dirección, administración, inspección y vigilancia, según el cual se desarrollan las competencias de las entidades territoriales en materia educativa. Así, en el artículo 150 se dispone simplemente que los concejos municipales "regulan la educación dentro de su jurisdicción".

Por otro lado, la Ley 715 de 2001 establece en el art. 7 las competencias de los municipios certificados en materia educativa, dentro del cual tiene -entre otras- la de "7.1. Dirigir, planificar y prestar el servicio educativo en los niveles de preescolar, básica y media, en condiciones de equidad, eficiencia y calidad, en los términos definidos en la presente ley". Así como "7.11. Promover la aplicación y ejecución de los planes de mejoramiento de la calidad en sus instituciones", y "7.12. Organizar la prestación del servicio educativo en su jurisdicción."

Dentro de este marco jurídico y en relación con la educación del Municipio de Medellín, el Concejo de la ciudad ha expedido múltiples normas en todos los campos posibles de la educación: la infraestructura, la denominación de los establecimientos educativos, auxilios y donaciones, dotación, materiales didácticos, alimentación para los estudiantes de escasos recursos, exenciones tributarias para estimular el apoyo a la educación, necesidades educativas especiales, primera infancia, educación ambiental, educación artística, recreación y deportes, beneficios a docentes, apoyo a la educación, entre otros.

Y obviamente, la perspectiva de género no ha sido ajena en los debates políticos y jurídicos que ha dado el Concejo del Municipio de Medellín en relación con la educación.

\section{BREVE RECUENTO ESTADÍSTICO HISTÓRICO DE LA EDUCACIÓN POR GÉNEROEN MEDELLÍN}

Haciendo la revisión histórica de la separación de género en la educación de la ciudad, se identifica que, según Agapito Betancur 
(2003), la primera escuela privada de niñas fundada en Medellín nació en 1832 dirigida por cuatro maestras. Y que para el año siguiente, 1833, otras señoras fundaron una nueva escuela con 40 alumnas (p. 37).

Destaca Betancur (2003) que en 1835 había en la Provincia 14 escuelas de niñas. En 1837 dos escuelas públicas y 17 privadas, con un total de 445 alumnas. En 1838 ya eran tres oficiales con 172 niñas y 25 privadas con 425 niñas. Hasta esta fecha, es clara la predominancia de entidades privadas en la educación femenina.

En 1839 había 16 escuelas públicas con 390 niñas. Y que el primer colegio (no escuela) de señoritas se abrió el 7 de abril de 1850, denominado "Santa Teresa de Jesús"(p.37).

También en el texto de Germán Hoyos Misas sobre Medellín, que data de 1916 pero fue reimpreso en el año 2004, se encuentran algunos datos relacionados con la educación por género. Sobre la instrucción pública, relata que en el Departamento se educaban en 1915, 40.907 varones y 38.987 mujeres con 1.358 maestros de ambos sexos en 883 escuelas. Como puede verse, en la educación pública, era relativamente baja la diferencia entre escolaridad de hombres y mujeres, participando ellas con casi el $49 \%$ de la población, lo que evidencia una aceptación general por la educación del sexo femenino en la región (p.47).

Respecto de la instrucción privada, comenta que había 1.189 varones y 1.423 mujeres en 33 escuelas alternadas, 10 urbanas (dos de varones y ocho de mujeres) y 35 escuelas rurales ( 3 de varones, 3 de mujeres y 29 alternadas) (p. 47). Como salta a la vista, en 1915, la educación de mujeres en el sector privado superaba a los hombres tanto en personal como en escuelas, lo que da cuenta de un reconocimiento, aunque relativo, de la conciencia sobre la importancia de la educación para las mujeres.

En otro apartado, el texto de Hoyos (2004) hace referencia a los establecimientos privados de instrucción secundaria y profesional del Departamento entre 1915 y 1916. Aquí, afirma que había matriculados 2.779 varones y 3.234 mujeres (p. 47) lo que de nuevo evidencia una preeminencia del género femenino en las aulas.

Además, existía el Colegio Central de Señoritas, que para 1916 contaba con 170 estudiantes que veían clases de fotograbado, mecanografía, 
contabilidad, idiomas, costura y modistería, música, arte culinario, así como higiene y medicina (p. 50).

Deja constancia Hoyos, que para ese año, el Instituto de Bellas Artes (hoy Fundación Universitaria Bellas Artes), fundado por la Sociedad de Mejoras Públicas, contaba con 59 varones y 37 señoritas (p. 50). Sobre la Normal de Institutoras dice que tenía 280 alumnas en 1915 y 116 niñas en la Escuela Anexa a la Normal de Señoritas (p. 52); todo esto, entre otras instituciones de prestigio, muchas de las cuales perduran hoy en día.

\section{REGULACIÓN DE GÉNERO EN LA EDUCACIÓN DE MEDELLÍN: ACUERDOS MUNICIPALES EN MATERIA DE EDUCACIÓN CON CONTENIDOS DE GÉNERO}

Revisando el reporte de acuerdos municipales en relación con la educación en la ciudad de Medellín, se observan varias normas que hacen referencia al carácter de género en la educación de la ciudad, los cuales se revisaron bajo tres categorías: 1) los acuerdos que aunque no hacen referencia a la educación hacen uso de un lenguaje incluyente para los menores de edad; 2) los que no hacen una referencia exclusiva a la educación pero incluyen la educación o la Secretaría de Educación desde una perspectiva de transversalidad; 3) los que versan sobre educación pero sin hacer alusión a una perspectiva de género usan un lenguaje incluyente, y 4) los que hacen expresa referencia a la educación de género.

Entre el periodo 1943-2000, solo se identifica un acuerdo municipal que haga uso del lengua diferencial en el tema educativo. Así, por Acuerdo Municipal 19 de 1961, se autoriza celebración de contrato para construir una escuela para niñas en el Barrio Villa Hermosa, situada en la carrera 39 con la calle 62. Y en 2001, se expide el Acuerdo 74, por medio del cual se reconoce y desarrolla la educación obligatoria para niños, niñas y jóvenes entre los 5 y los 15 años de edad, comprendiendo desde el preescolar hasta el grado $9^{\circ}$. Como se observa en la anterior disposición, para el año 2001 se incluye el lenguaje diferencia para uno y otro género.

Pero se destaca que revisado el periodo 1943-2012, el lenguaje inclusivo empieza a percibirse de manera continua en los acuerdos municipales en materia de educación a partir del año 2005. 
Así, se encuentra el Acuerdo 23 de 2005, en el cual se establece una política pública para la detección y atención integral a niños con capacidades excepcionales en Medellín y sus cinco corregimientos.

Para el año siguiente, la Corporación Municipal expide el Acuerdo 09 de 2006, "Por el cual se establece una política pública para la Prevención y Atención de las violencias sexuales que afectan a la ciudadanía, principalmente a mujeres, niñas y niños en la ciudad de Medellín", que si bien no hace referencia alguna al tema educativo, establece en el art. 4 que la Secretaría de Educación, junto con las Secretarías de Salud, Cultura Ciudadana y Gobierno, bajo la dirección de la Secretaría de Inclusión Social, conformarán el Comité Coordinador Municipal para la Prevención y Atención de las Violencias Sexuales, que funge como el organismo articulador de la política pública.

En esta misma línea, sin referencia exclusiva a la educación pero bajo el lenguaje incluyente de género, está el Acuerdo Municipal 50 de 2009, "por medio del cual se establecen medidas tendentes a la prevención y sanción del abuso y la explotación sexual de niños, niñas y adolescentes en el municipio de Medellín", el cual restringe el ingreso de niños, niñas y adolescentes a los establecimientos donde se preste comercialmente el servicio de internet a través de cabinas o espacios privados del Municipio de Medellín, cuando no estén acompañados de sus padres, representantes legales o adultos responsables autorizados, con las excepciones de las salas de internet administradas por bibliotecas públicas y establecimientos educativos.

De igual modo, el Acuerdo 27 de 2009 establece en el Concejo de Medellín el día de los niños, niñas y adolescentes concejales a cargo de la Mesa Directiva del Concejo y de la Secretaría de Educación; el cual se destaca por el lenguaje incluyente más que por su relación específica en materia educativa.

El lenguaje diferencial se observa también en el Programa Institucional y Municipal de Ferias de la Ciencia, la Tecnología y la Innovación, cuyo objetivo general es aportar en la construcción de una cultura que tenga a la ciencia, la tecnología y la innovación como componentes importantes del desarrollo social mediante la participación de los niños, niñas y jóvenes -estudiantes de educación básica y media- en procesos de investigación que se inician desde el aula de clase (Acuerdo 85 de 2009).

Para inicios de 2010, se encuentra el concepto $\mathrm{N}^{\circ} 22$ del 23 de marzo, Rad. 201000109995, en el cual la Secretaría General remite a la 
Comisión Tercera del Concejo, un concepto jurídico sobre la viabilidad de un proyecto por el cual se adoptaría una política pública para la transversalización del enfoque de género en la educación, enumera las actividades que la Secretaría de Educación ya venía realizando desde esta perspectiva, entre las que se contaban la capacitación de 72 docentes con el Ministerio de Educación sobre educación sexual y construcción de ciudadanía; talleres para los docentes realizados por la Secretaría de Bienestar Social sobre diversidad sexual, que incluían cuatro revistas ilustradas y una guía metodológica; el programa " $l a$ escuela busca la mujer adulta"; un diplomado en género dictado por la FLACSO de Argentina para docentes y funcionarios del municipio y sus entidades descentralizadas y otro diplomado en género y educación.

En materia de primera infancia, igualmente se identifican ambos géneros, al autorizar al Alcalde de Medellín para comprometer vigencias futuras (Buen Comienzo), para los proyectos: "Niños y niñas 0-12 meses Sisbén I y II", "Educación inicial de 1 a 4 años" y "Complementación alimentaria primera Infancia" (Acuerdo 19 de 2010).

Paro el año 2011 se encuentran tres acuerdos municipales que van más allá del mero lenguaje diferencial para adoptar políticas públicas concretas en materia de género. Es el caso de los Acuerdos 8, 20 y 36.

A través del Acuerdo 8, se adopta la política pública para el reconocimiento de la diversidad sexual e identidades de género y para la protección, restablecimiento, atención y la garantía de derechos de las personas lesbianas, gais, bisexuales, transgeneristas e intersexuales (LGBTI) del Municipio de Medellín.

Por Acuerdo 20 de 2011, se ajusta la política de Prevención y Atención de las violencias sexuales que afectan a la ciudadanía, principalmente a mujeres, niñas, niños y adolescentes en el Municipio de Medellín.

Y en el marco de la Política Pública para la transversalización del enfoque de género en los Proyectos Educativos Institucionales de las Instituciones Educativas de la ciudad de Medellín, se expide el Acuerdo 36 de 2011, en el cual se ordena a la Secretaría de Educación incluir algunos indicadores de equidad de género en los criterios de calificación del premio "Medellín la más educada" y en otros premios de calidad o concursos que se promuevan con las Instituciones Educativas oficiales de Medellín, como una forma de estimular el cumplimiento de la 
presente Política Pública, por parte de las Instituciones Educativas (Acuerdo 36/11).

Este acuerdo, con un claro enfoque educativo, plantea como objetivo de la política pública, que en la práctica pedagógica cotidiana, del día a día en las instituciones educativas, se favorezca la inclusión del enfoque de género aprovechando que estos son espacios de socialización de niños, niñas y adolescentes, donde se formen como ciudadanos y ciudadanas con una mentalidad de justicia e inclusión, con igualdad de oportunidades para hombres y mujeres en todas las etapas de la vida (art. 2).

Para lo anterior, el acuerdo municipal encarga de esta responsabilidad a la Secretaría de las Mujeres (creada en el año 2007) y a la Secretaría de Educación, y les asigna varias obligaciones: crear programas de sensibilización y formación docente (art. 3); promover y apoyar la elaboración de material didáctico con enfoque de género (art. 5); a las instituciones educativas las obliga a incluir en sus proyectos educativos institucionales el enfoque de género (art. 7) y un índice de equidad de género (art. 8); también dispone que las instituciones educativas deben estimular a los docentes de todas las áreas del conocimiento "para que incluyan en sus respectivas asignaturas, el estudio y la visibilización de las mujeres, tanto en las elaboraciones conceptuales, como en la construcción de la historia de la humanidad" (art. 10).

Así mismo, la Secretaría de Educación debía incluir "algunos indicadores de equidad de género en los criterios de calificación del premio 'Medellín la más educada' (...) como una forma de estimular el cumplimiento de la presente Política Pública, por parte de las Instituciones Educativas" (art. 6); promover la utilización de lenguaje no sexista (art. 9) y visibilizar las experiencias exitosas de las prácticas pedagógicas no sexistas (art.11).

\section{CONCLUSIONES}

Se concluye que en el orden nacional hay una exhortación a la educación mixta, en aras de formar en la equidad de género, pero no hay normas que prohíban o exijan que los establecimientos educativos deban tener una población femenina, masculina o mixta, situación que dependerá de la autonomía de las instituciones de educación y su proyecto educativo institucional. 
No obstante, en general, puede evidenciarse la clara tendencia al reconocimiento de la diversidad en cuanto al género y su integración en el sistema educativo desde el mismo sistema jurídico nacional, protegiendo especialmente las mujeres cabeza de familia y víctimas de la violencia.

En este marco se ha establecido el derecho a la educación de las madres gestantes, situación que obliga, incluso, a modificar los manuales de convivencia cuando van en contravía del derecho a la maternidad y educación, con indiferencia de si son instituciones públicas, privadas, confesionales o no.

De igual modo, los manuales de convivencia y la comunidad educativa deben garantizar la protección y el reconocimiento a la identidad y orientación sexual, toda vez que ellos forman parte del núcleo esencial de la dignidad, la libertad y la autonomía de toda persona.

Respecto de los acuerdos municipales expedidos en el Municipio de Medellín en materia de educación y género, se observó que entre el periodo de 1943 a 2000 solo se identifica uno solo. Pero a partir del año 2005 se expiden varios acuerdos.

Revisando el reporte de acuerdos municipales en relación con la educación en la ciudad de Medellín, se identifican varias normas que hacen referencia al carácter de género en la educación de la ciudad, los cuales se revisaron bajo tres categorías: 1) los acuerdos que aunque no hacen referencia a la educación hacen uso de un lenguaje incluyente para los menores de edad; 2) los que no hacen una referencia exclusiva a la educación pero incluyen la educación o la Secretaría de Educación desde una perspectiva de transversalidad; 3) los que versan sobre educación pero sin hacer alusión a una perspectiva de género usan un lenguaje incluyente, y 4) los que hacen expresa referencia a la educación de género.

Concretamente en el año 2011 se expiden tres acuerdos municipales relacionados con la respeto por la diversidad de género, entre los que se destaca el Acuerdo 36 de 2011 sobre transversalización de género en la educación.

Con todo esto se evidencia el interés de la Corporación Municipal por regular el tema en la ciudad, pero además en las instituciones educativas, buscando una educación inclusiva, respetuosa, pacífica. 


\section{REFERENCIAS BIBLIOGRÁFICAS}

Castillo Sánchez, M. y Gamboa Araya, R. (2013). La vinculación de la educación y género. Revista Electrónica "Actualidades Investigativas en Educación", 13(1), p. 1-16.

Chavarría Serna J. M. (2014). La instrucción pública primaria en Medellín durante la regeneración 1886-1899. Medellín: Universidad Nacional de Colombia.

Colombia. Concejo de Medellín. (1961). Acuerdo 19.

Colombia. Concejo de Medellín. (2001). Acuerdo 74.

Colombia. Concejo de Medellín. (2005). Acuerdo 23.

Colombia. Concejo de Medellín. (2006). Acuerdo 09.

Colombia. Concejo de Medellín. (2009). Acuerdo 27.

Colombia. Concejo de Medellín. (2009). Acuerdo 50.

Colombia. Concejo de Medellín. (2009). Acuerdo 85.

Colombia. Concejo de Medellín. (2010). Acuerdo 19.

Colombia. Concejo de Medellín. (2011). Acuerdo 08.

Colombia. Concejo de Medellín. (2011). Acuerdo 20.

Colombia. Concejo de Medellín. (2011). Acuerdo 36.

Colombia. Corte Constitucional. (1992). Sentencia T-440/92. Magistrado Ponente: Eduardo Cifuentes Muñoz.

Colombia. Corte Constitucional. (1996). Sentencia T-290/96. Magistrado Ponente: Jorge Arango Mejía.

Colombia. Corte Constitucional. (1998). Sentencia SU-641/98. Magistrado Ponente: Carlos Gaviria Díaz. 
Colombia. Corte Constitucional. (1998). Sentencia T-101/98. Magistrado Ponente: Fabio Morón Díaz.

Colombia. Corte Constitucional. (1998). Sentencia T-656/98. Magistrado Ponente: Eduardo Cifuentes Muñoz.

Colombia. Corte Constitucional. (1999). Sentencia SU-337/99. Magistrado Ponente: Alejandro Martínez Caballero.

Colombia. Corte Constitucional. (1999). Sentencia T-412/99. Magistrado Ponente: Antonio Barrera Carbonell.

Colombia. Corte Constitucional. (2004). Sentencia T-220/04. Magistrado Ponente: Eduardo Montealegre Lynett.

Colombia. Corte Constitucional. (2007). Sentencia T-458/07. Magistrado Ponente: Álvaro Tafur Galvis.

Colombia. Corte Constitucional. (2009). Sentencia T-393/09. Magistrado Ponente: Nilson Pinilla Pinilla.

Colombia. Corte Constitucional. (2009). Sentencia T-396/99. Magistrado Ponente: Eduardo Cifuentes Muñoz.

Colombia. Corte Constitucional. (2009). Sentencia T-564/99. Magistrado Ponente:Alfredo Beltrán Sierra.

Colombia. Corte Constitucional. (2011). Sentencia T-062/11. Magistrado Ponente: Luis Ernesto Vargas Silva.

Colombia. Corte Constitucional. (2012). Sentencia C-682/12. Magistrado Ponente: Nilson Pinilla Pinilla.

Colombia. Corte Constitucional. (2013). Sentencia T-565/13. Magistrado Sustanciador: Luis Ernesto Vargas Silva.

Colombia. Corte Constitucional. (2013). Sentencia T-603/13. Magistrado Ponente: Jorge Iván Palacio Palacio.

Colombia. Instituto Colombiano de Normas Técnicas y Certificación (1999). Norma Técnica Colombiana NTC 4595 sobre Planeamiento y Diseño de Instalaciones y Ambientes Escolares. Medellín: 


\section{ICONTEC.}

Colombia. Ministerio de Educación Nacional (1993). Resolución 3353 de 1993.

Colombia. Presidencia de la República (1886). Decreto 595.

Colombia. Presidencia de la República (1893). Decreto 429.

Colombia. Presidencia de la República (1894). Decreto 001.

Colombia. Presidencia de la República (1904). Decreto 491.

Colombia. Presidencia de la República (1994). Decreto 1860.

Díaz-Aguado Jalón, M.J y Martín G. (2011). Convivencia y aprendizaje escolar en la adolescencia desde una perspectiva de género. Psicothema. Vol. 23, nº 2, p. 252-259.

Hoyos Misas, G. (2004). Guía ilustrada de Medellín. Medellín: Instituto Tecnológico Metropolitano.

Instituto Colombiano de Normas Técnicas y Certificación (1999). Norma Técnica Colombiana NTC 4595 sobre Planeamiento y Diseño de Instalaciones y Ambientes Escolares. Medellín: ICONTEC.

Martínez Sampere, E. (2004). La Constitución y la educación mixta igualitaria. Revista de Educación, (6), p. 55-64. 